\title{
CrystEngComm
}

Check for updates

Cite this: CrystEngComm, 2021, 23, 5925

Received 6th April 2021,

Accepted 11th July 2021

DOI: $10.1039 /$ d1ce00462j

rsc.li/crystengcomm

\section{A high contrast mechanochromic luminescent diacetylene-linked bis-benzothiadiazole derivative $\uparrow$}

\author{
Marcelo Echeverri, (DD Constanza Ruiz and Berta Gómez-Lor (iD*
}

\begin{abstract}
Two new rod-shaped molecules based on a directly-linked or a diacetylene-linked bis-benzothiadiazole core flanked by two $p$-nonylphenyl groups have been synthesized and their potential stimuli-responsive behaviour was investigated. The high molecular anisotropy and the presence of flexible chains induce these molecules to organize into lamellar self-assemblies, which can be influenced by the action of external stimuli. The two compounds present a polymorphic transformation upon heating which can be reversed upon shearing, but it is only in the diacetylene linked derivative that this transformation is accompanied by a change of its emission colour. In fact, in this material, the phase interconversion involves a high contrast luminescence alteration which can be rationalized considering the high conformational flexibility of the bridging unit which offers a great opportunity to change the surrounding of the molecules in the solid upon minor stimuli. Conversely, in the directly connected bis-benzothiadiazole, the molecules are closely packed in the layers, through cooperative chalcogen and aromatic interactions, probably preventing the displacement of the molecules within the layers and therefore changes in the environment of their aromatic parts responsible for the colour and emissive properties of the solids. The results of this study point to the flexible diacetylene moiety as an attractive design element in the construction of stimuli responsible light emitting materials. We hope that this observation will facilitate the design of new mechanochromic materials in the near future.
\end{abstract}

\section{Introduction}

Mechanochromic materials, that is, solids possessing optical properties that vary upon mechanical stimulation, arouse much interest for rewritable paper, ${ }^{1}$ recording ${ }^{2}$ and stress sensing. ${ }^{3}$ Many organic ${ }^{4,5}$ and organometallic compounds ${ }^{6}$ have been recently reported whose color or luminescence properties are altered upon grinding, pressing or stretching. The origin of this interesting phenomenon can be usually found in the high dependence of the optical properties of molecular materials on their surroundings. ${ }^{7,8}$ This high dependence offers many opportunities to synthesize materials which can respond to external stimuli by varying their supramolecular arrangements, however, only in a few examples, a precise understanding about the origin of the colour change has been achieved. Single crystal diffraction analysis is probably the most powerful tool to attain such understanding, as it provides accurate information on the

Instituto de Ciencia de Materiales de Madrid, CSIC Cantoblanco, 28049, Madrid, Spain.E-mail: bgl@icmm.csic.es

$\dagger$ Electronic supplementary information (ESI) available: General information, synthesis and characterization of $3-5$, copy of the ${ }^{1} \mathrm{H}$ NMR and ${ }^{13} \mathrm{C}$ NMR spectra, single crystal X-ray structure determination and PXRD patterns of 5. Crystal data in CIF of 4 CCDC 2074632. For ESI and crystallographic data in CIF or other electronic format see DOI: 10.1039/d1ce00462j relative arrangements of neighbouring molecules and the interactions involved among them. ${ }^{9,10}$ However, although there are remarkable examples in the literature of molecular materials that retain their crystal integrity after a mechanical operation, ${ }^{11-14}$ the emission switching between polymorphs is rather exceptional as it involves the transformation of the molecular structure in the constrained environment of the crystal. Notwithstanding, some design hints have been already proposed to induce a mechanochromic behaviour ${ }^{15,16}$ among them the presence of weak interaction planes which allow the relative slippage of the molecules affecting the interactions with their next neighbours, and consequently their optical properties. ${ }^{17-19}$ However, despite the impressive progress of this field in recent years, to date the discovery of new mechanochromic compounds is still largely based on serendipity.

In the quest for new organic stimuli responsive materials, the 2,1,3-benzothiadiazole moiety (BTD) has emerged as a promising building block, as the absorption and emission properties of its derivatives are strongly influenced by their external environments. ${ }^{20,21}$ In the last few years, several BTDbased chromogenic and fluorogenic materials have been reported, but although there are some recent exceptions, ${ }^{19,22,23}$ in most examples, crystalline-to-amorphous transitions were the origin of this behaviour. ${ }^{20,24-28}$ This fact 
prevents an in-depth understanding of the mechanism behind the different optical properties observed. We have recently reported on a BTD-derivative which crystallizes in two distinctive luminescent crystal phases (blue and green emitting) which can be reversibly switched by the action of pressure and solvent vapours. In this molecule, the BTD moiety is flanked by two phenyl groups substituted with flexible nonyl chains, which induce the assembly of these molecules into layers and provide weak interaction planes which are presumably responsible for the switchable properties by facilitating mechanically induced sliding processes and provoking the displacement of the molecules with respect to the layer normal. ${ }^{19}$

In this work, we have explored the possibility of extending this design principle to obtain BTD-based mechanochromic materials with larger contrast switchable emission colours. To this end, we have synthesized two different rod-shaped BTD-dimeric molecules (with the two BTD subunits either directly linked or separated by a diacetylene bridge) flanked by two 4-nonylphenyl groups. By dimerizing the BTD molecule, we could significantly red-shift the absorption and emission properties of these materials in the solid state while the presence of flexible chains induces their self-assembly into layers thus providing weak interaction planes. Interestingly, although in the two cases a polymorphic transformation can be observed upon shearing, it is only in the case of the diacetylene-linked derivative that a change of colour can be observed.

\section{Results and discussion}

\section{Synthesis and characterization}

The synthesis of the dimeric compounds $\mathbf{4}$ and $\mathbf{5}$ was performed starting from 4-bromo-7-(4-nonylphenyl)benzo[c][1,2,5] thiadiazole 2 which can be readily obtained starting from commercial 4,7-dibromo-benzo[c] [1,2,5]thiadiazole 2 through
Suzuki coupling with one equivalent of 4-nonylphenylboronic acid as has been previously reported. ${ }^{22}$

A self-coupling reaction of 2 catalysed by $\mathrm{Pd}(\mathrm{OAc})_{2}$ in polyethylene glycol (PEG4000) ${ }^{29,30}$ rendered straightforwardly compound 4 in good yield (Scheme 1). Compound 5 was obtained by $\mathrm{Cu}$-catalysed oxidative Eglinton coupling of the corresponding ethynyl derivative 3 , which is readily accessed in two steps by Sonogashira coupling of 2 with ethynyl trimethylsilane, followed by deprotection with KF (Scheme 1).

All new compounds were purified by silica gel column chromatography, using a mixture of hexane/ $\mathrm{CH}_{2} \mathrm{Cl}_{2}(3: 1)$ as eluent and characterized by ${ }^{1} \mathrm{H}$ NMR, ${ }^{13} \mathrm{C}$ NMR, and mass spectrometry (see the ESI $\dagger$ for experimental details).

The photophysical properties of the new molecules were studied by UV-vis and fluorescence spectroscopy. The UV-vis absorption spectra of $\mathbf{4}$ and $\mathbf{5}$ in $\mathrm{CH}_{2} \mathrm{Cl}_{2}$ exhibit one absorption band, localized around $280 \mathrm{~nm}$ (associated to $\pi-\pi$ transition), and a lower energy band around $400 \mathrm{~nm}$ (see Fig. 1a) which can be ascribed to charge transfer transitions. Both bands are slightly red-shifted when going from 4 to 5 in agreement with a better electron delocalization facilitated by the diacetylene linker. The fluorescence spectra of both compounds in $\mathrm{CH}_{2} \mathrm{Cl}_{2}$ are characterized by an emission band peaking at 511 and $499 \mathrm{~nm}$ for 4 and 5, respectively (Fig. 1a).

All compounds can be easily reduced to stable radical anions reflecting the strong acceptor character of the BTD core. In both derivatives, it is possible to observe two reversible one electron reduction waves, indicating that the first reduction yields a monoanion stabilized by delocalization over both electron accepting units, and confirming the good electronic communication between the two BTD moieties. The tworeduction waves of $\mathbf{5}$ are slightly shifted anodically as compared to 4 suggesting that the diacetylene bridge renders the system more electron deficient. Interestingly compound $\mathbf{4}$ presents an amphoteric redox character as it can be also easily oxidized in the accessible solvent window (Fig. 1b).

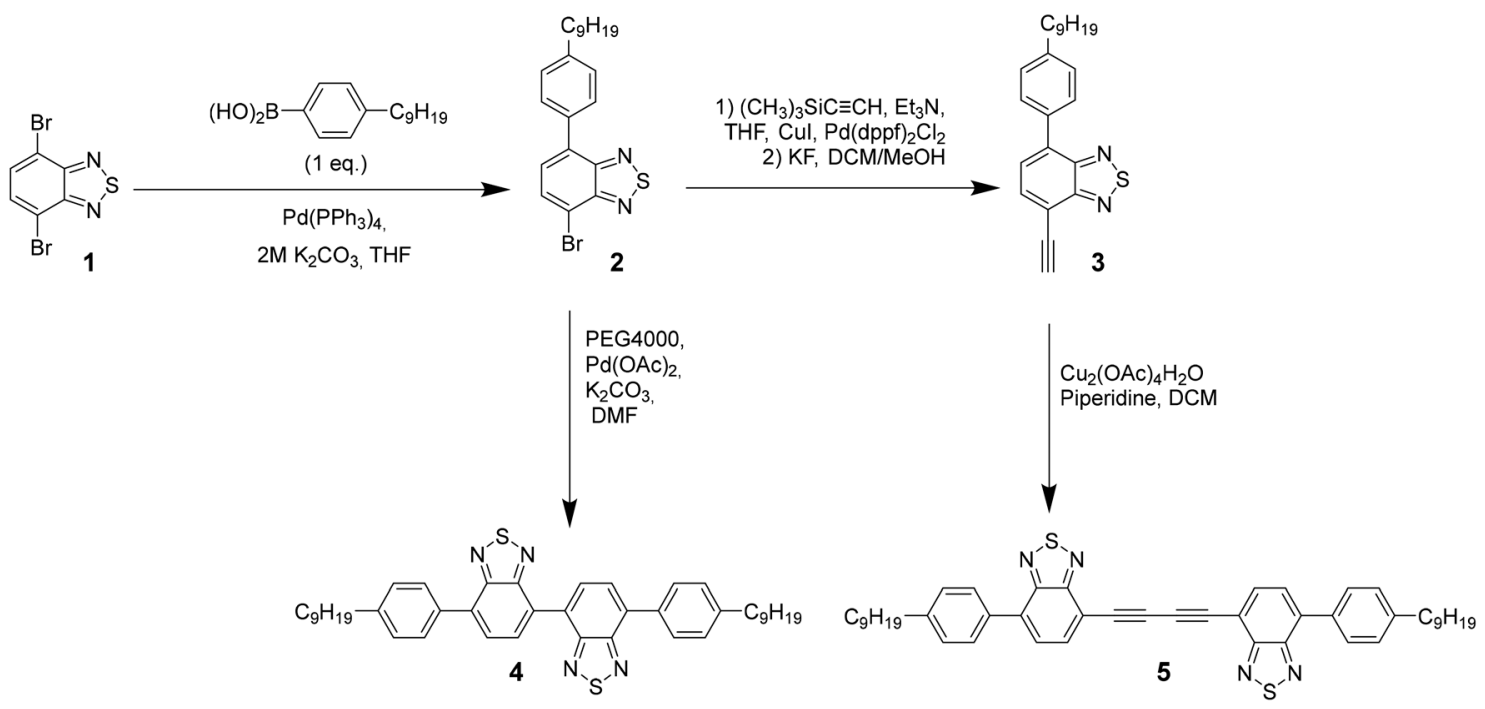

Scheme 1 The synthesis of compounds 1-5. 


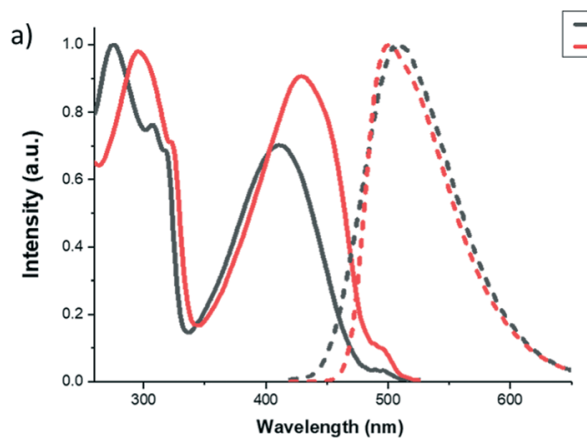

b)

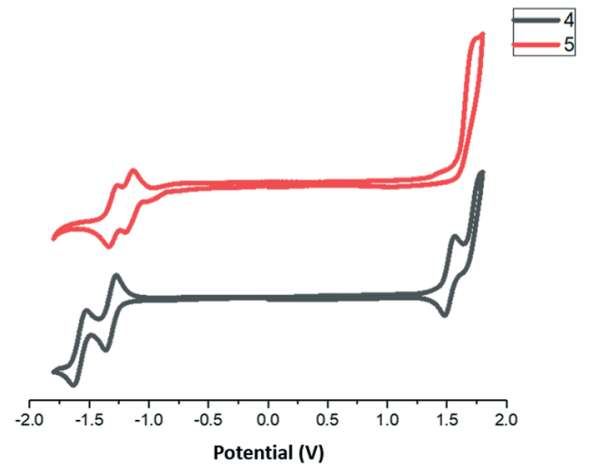

Fig. 1 a) Normalized UV-vis (lines) and fluorescence (dots) spectra of compounds 4-5 in $\mathrm{CH}_{2} \mathrm{Cl}_{2}, \mathrm{C}=5 \times 10^{-6} \mathrm{M}$ solutions. b) Cyclic voltammograms of compounds 4-5 at $[\mathrm{C}]=1 \times 10^{-3} \mathrm{M}$ recorded at a scan rate of $100 \mathrm{mV} \mathrm{s}^{-1}$ in $\mathrm{CH}_{2} \mathrm{Cl}_{2} / 0.1 \mathrm{M} \mathrm{TBAPF}_{6}$ measured versus $\mathrm{Ag}$ / $\mathrm{AgCl}$.

\section{Crystal growth and structure determination of 4}

In order to shed light on the influence of the bridging mode on the supramolecular arrangements of these molecules, we have attempted to grow single crystals of both compounds. Yellow single crystals suitable for X-ray structure determination of $\mathbf{4}$ were obtained by slow evaporation of a $\mathrm{CH}_{2} \mathrm{Cl}_{2}$ solution. Compound $\mathbf{4}$ crystalized in the triclinic space group $P \overline{1}$, with five crystallographically independent molecules in the asymmetric unit cell (see the ESI† Fig. S7), which differ slightly in the torsion between the two BTD moieties (situated anti to each other with dihedral angles of 36.9-37.8 $)$ and between the external phenyl rings and the neighbouring BTD (with torsion angles that vary from 34.0 and 40.2). The crystal packing presents a lamellar organization with no interdigitation of alkyl chains, confirming in this case the presence of weak interaction planes (Fig. 2a).

Within the layers, each independent molecule interacts with its symmetry-related molecules forming sheets that grow along the a direction. In the sheets, each molecule is connected with its two next neighbours through chalcogen bonding $2 \mathrm{~S}-2 \mathrm{~N}$ squares. As a result, all the thiadiazole rings are involved in this type of interaction. Please note that the highly directional $2 \mathrm{~S}-2 \mathrm{~N}$ square motif ${ }^{31}$ has emerged as a powerful synthon in supramolecular chemistry and crystal engineering. ${ }^{32}$ These sheets are further connected along the $c$ direction through the cooperative action of a number of S-N, $\mathrm{CH}-\pi$ and S- $\pi$ interactions (Fig. 3 ). ${ }^{33}$ a)

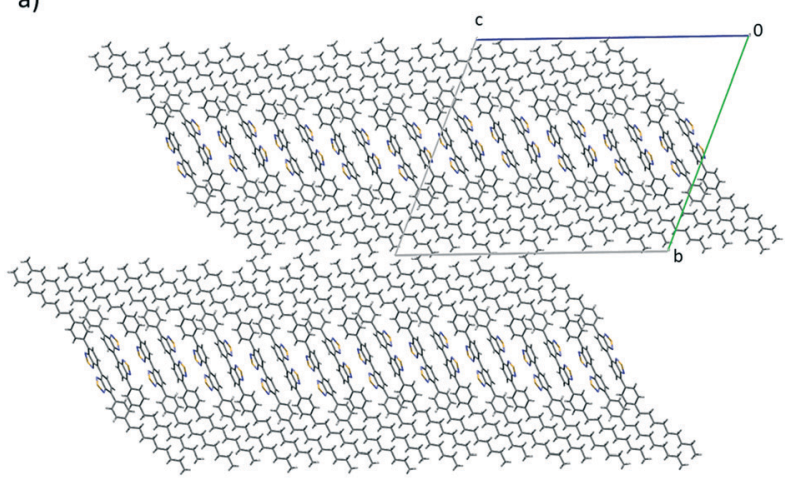

b)

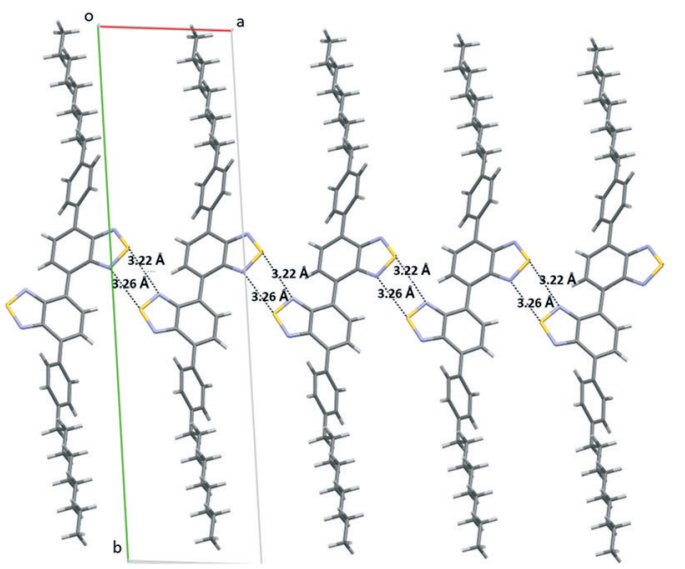

Fig. 2 a) A view of the crystallographic packing of 4 showing the layered arrangement. b) A view of the sheets that form the layer growing along the $a$ direction interacting through chalcogen bonding $2 \mathrm{~S}-2 \mathrm{~N}$ squares.

Conversely all attempts to obtain crystals of $\mathbf{5}$ of sufficient quality for structure determination by slow evaporation of a variety of pure solvents and different solvent mixtures were unfruitful. However, these experiments confirmed the existence of two polymorphs with a similar yellowish colour under visible light but which can be easily differentiated upon irradiation with a $365 \mathrm{~nm}$ lamp. Thus evaporation from

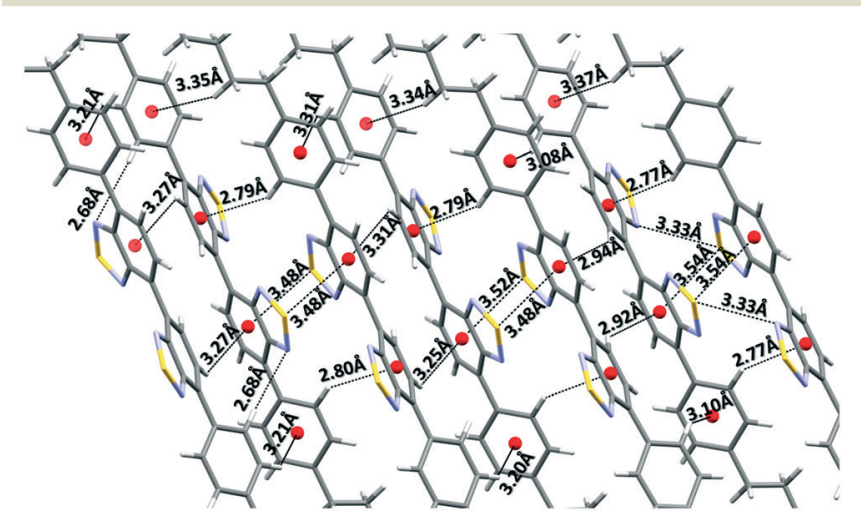

Fig. 3 Depiction of the close cooperative network of interactions among the sheets in the $c$ direction. 
toluene led to yellow emitting plate-like crystals, while slow evaporation of a $\mathrm{CH}_{2} \mathrm{Cl}_{2}$ : propanol mixture gave rise to orange-emitting ribbon-like crystals, with highly anisotropic shapes that bend and twist as they grow, giving rise in some cases to curious helicoidal morphologies (Fig. 4c and d). This behaviour has been commonly observed in crystals with a lamellar packing. ${ }^{34}$ Unfortunately these crystals in spite of having the appearance of single crystals diffract poorly and their crystal structure could not be solved.

X-ray powder diffractograms of both polymorphs show a similar packing arrangement characterized by major primary diffraction peaks at $3.17^{\circ}$ and $2.83^{\circ}$ for the yellow and the orange polymorphs, respectively (see the ESI, $\uparrow$ Fig. S8 and S9), which agrees with lamellar structures with interlayer distances of $27.8 \AA$ and $31.2 \AA$. Fig. 5 shows the absorption and emission spectra of compound 4 and polymorphs $5 \alpha$ and $5 \boldsymbol{\beta}$. As can be observed in Fig. 5, the absorption spectra of the three solids are very similar, however, while the emission spectra of 4 and polymorph $5 \alpha\left(\lambda_{\max }=525 \mathrm{~nm}\right)$ also coincide, the emission spectrum of polymorph $\mathbf{5 \beta}$ is redshifted by $80 \mathrm{~nm}\left(\lambda_{\max }=605 \mathrm{~nm}\right)$.

\section{Polymorphic transformation and mechanochromic/ thermochromic behaviour}

The lamellar organization observed in both compounds (and therefore the presence of weak interaction planes in the crystals) together with the evidence of the existence of at least two polymorphs of $\mathbf{5}$, induced us to evaluate in more detail the possible polymorphic transformation by exposing them to different physical stimuli.

Thus, thermal treatment of $\mathbf{4}$ produced a polymorphic transformation at $70{ }^{\circ} \mathrm{C}$ as could be monitored by powder X-ray diffraction (Fig. 6). This transformation is not reverted upon cooling, but shearing the cooled sample with a spatula gives rise again to the initial phase. X-ray powder diffractograms of both polymorphs are dominated by primary diffraction peaks at $2.84^{\circ}$ (as obtained) and $2.94^{\circ}$ (after a heating-cooling cycle), which suggests that the lamellar packing is maintained after the thermal treatment although they differ slightly in the interlayer distance. Heating a single crystal of the as-obtained polymorph under a polarizing microscope (POM) equipped with a hot-stage allowed us to visualize the progressive changes in the birefringence that
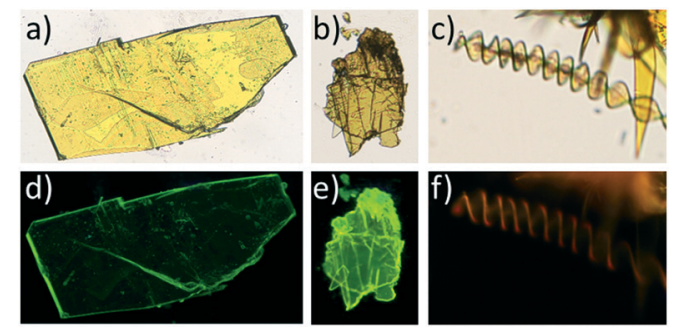

Fig. 4 Optical microscopy images of 4 (a), $5 \alpha$ (b) and $5 \beta$ (c) viewed under visible light and under UV-light emission (4 (d), $5 \alpha$ (e) and $5 \beta$ (f)).

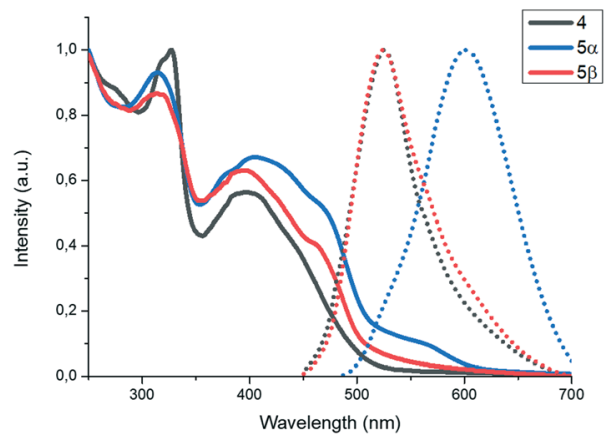

Fig. 5 Normalized solid UV-vis (lines) and fluorescence (dots) spectra of compound 4 and polymorphs $5 \alpha$ and $5 \beta$.

accompanies this phase transformation. In this case, although the outer shape of the single crystal is maintained, its integrity is lost as evidenced by the emergence of numerous cracks in its surface.

Curiously, in this case, the polymorphic transformation is not accompanied by a colour or luminescence alteration. This behaviour suggests that although the presence of weak interaction planes allows some layer sliding, upon external stimulation, the compact network of intermolecular interactions that connect the molecules within the layers maintains the relative positions of the aromatic moieties unaltered (see Fig. 6a).

Both polymorphs of compound $\mathbf{5}$ were subjected to similar thermal and shearing treatments, which allowed us to confirm that both polymorphs can be interconverted. Thus, heating the orange emitting polymorph $5 \alpha$ at $120{ }^{\circ} \mathrm{C}$ gives rise to polymorph $\mathbf{5 \beta}$ as could be confirmed by powder X-ray

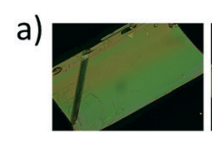

5 seconds )

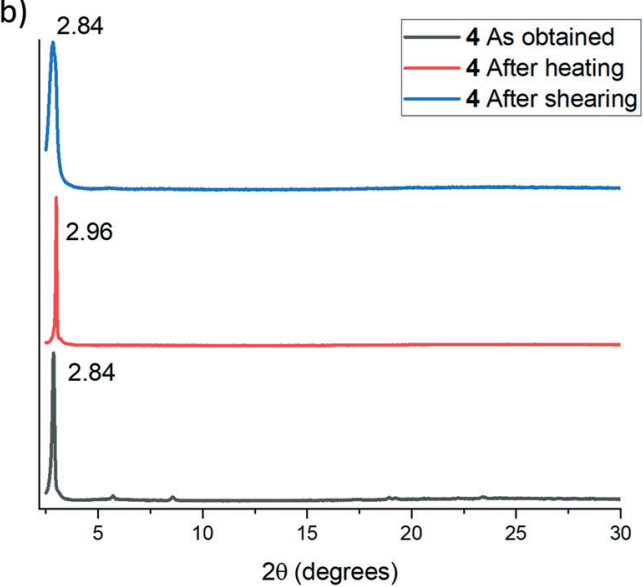

Fig. 6 a) POM images of the polymorphic transformation occurring on a single crystal at $70{ }^{\circ} \mathrm{C}$. b) Thermal and subsequent mechanochromic polymorphic transformations followed by powder $\mathrm{X}$-ray diffraction pattern changes. 
a)
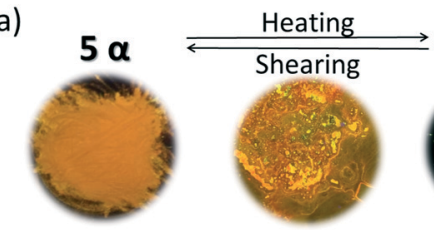

b)

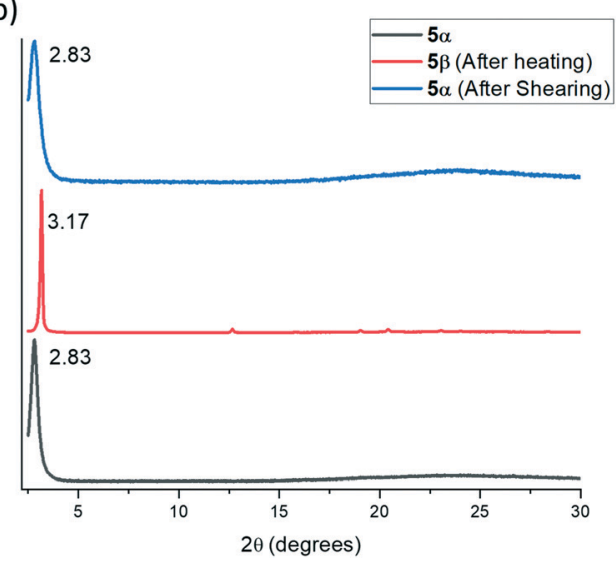

Fig. 7 (a) Photographs of the interconversion between polymorphs $5 \alpha$ and $5 \beta$ illuminated with a $365 \mathrm{~nm}$ lamp. (b) Thermal and subsequent mechanochromic polymorphic transformations followed by powder $\mathrm{X}$-ray diffraction pattern changes.
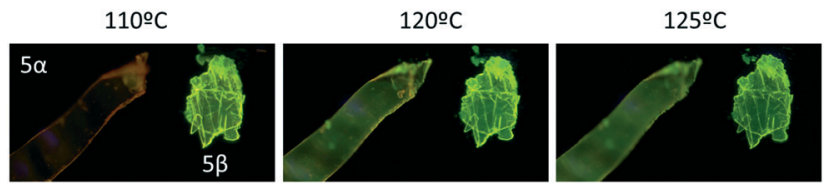

Fig. 8 Photomicrographs of the transformation of $5 \alpha$ into $5 \beta$ upon thermal heating illuminated with a $365 \mathrm{~nm}$ lamp.

diffraction. As previously observed for compound 4, this transformation is not reverted upon cooling. On the other hand, shearing the yellow emitting polymorph $5 \boldsymbol{\beta}$ (obtained after the thermal treatment) at room temperature gives rise again to the orange polymorph (Fig. 7). These easy transformations can be understood considering the high flexibility and low rotational barrier imposed by the diacetylene linker which allows for the easy modulation of the local geometry of the molecules and intermolecular interactions in the crystal, with evident influence on their optical properties. The reversible switching is maintained at least for 10 cycles, which, together with the high degree of contrast in the emitting colour between the two polymorphs, confers this material much interest in the development of rewritable paper ${ }^{1}$ or anticounterfeiting solutions. ${ }^{35}$

Interestingly the thermal phase transformation could be visualized on isolated crystals under a microscope as evidenced by the progressive change in the emission colour upon heating above $110{ }^{\circ} \mathrm{C}$ under illumination with a $365 \mathrm{~nm}$ lamp. In this case, no cracking was observed, confirming the higher elasticity of the assembly in this case, again in close relation with the flexibility provided by the diacetylene linker (Fig. 8).

\section{Conclusions}

Two new rod-shaped molecules based on a directly-linked or a diacetylene-linked bis-benzothiadiazole core flanked by two $p$-nonylphenyl groups have been synthesized and their potential mechanochromic behaviour was investigated. The high molecular anisotropy and the presence of flexible chains induce the self-assembly of these molecules into layers thus providing weak interaction planes, which has been previously found to be an attractive design principle to obtain stimuli responsive materials.

Indeed, in both materials, a reversible polymorphic transformation could be observed, which confirms the validity of this approach, although only in one of them, the transformation is accompanied by a variation in their optical properties. The different behaviour can be ascribed to the different linkages between the BTD moieties which influences the flexibility of the molecules and also how the molecules interact with each other in the solids after mechanically or thermally induced sliding processes. Thus, in the directly connected bis-BTD derivative, the molecules are involved in a cooperative network of intermolecular interactions which probably hinders the displacement of the molecules within the layers, while in the diacetylene-bridged derivative, the flexibility of the linker provides multiple easily accessible conformations which will influence how the molecules interact with each other in the solid. In fact, in this material, the phase interconversion involves a high contrast luminescence alteration (with a variation of its maximum emission wavelength of $80 \mathrm{~nm}$ ). Such behaviour confers this material much interest towards its potential applications and points to the flexible diacetylene moiety as an attractive design element in the construction of stimuli responsible light emitting materials.

The result of this study indicates that the presence of weak interaction planes is not sufficient to obtain BTD-based mechanochromic materials, but also loosely bound molecules within the layers will be required.

\section{Conflicts of interest}

There are no conflicts to declare.

\section{Acknowledgements}

This work was financially supported by the MICINN (PID2019104125RB-I00) of Spain. We gratefully acknowledge Dr Josefina Perles for solving the crystal structure of 4 .

\section{Notes and references}

1 M. I. Khazi, W. Jeong and J.-M. Kim, Adv. Mater., 2018, 30, 1705310 .

2 D. Genovese, A. Aliprandi, E. A. Prasetyanto, M. Mauro, M. Hirtz, H. Fuchs, Y. Fujita, H. Uji-I, S. Lebedkin, M. Kappes and L. De Cola, Adv. Funct. Mater., 2016, 26, 5271-5278.

3 D. R. T. Roberts and S. J. Holder, J. Mater. Chem., 2011, 21, 8256-8268. 
4 Y. Sagara, S. Yamane, M. Mitani, C. Weder and T. Kato, Adv. Mater., 2016, 28, 1073-1095.

5 Z. Chi, X. Zhang, B. Xu, X. Zhou, C. Ma, Y. Zhang, S. Liu and J. Xu, Chem. Soc. Rev., 2012, 41, 3878-3896.

6 X. Zhang, Z. Chi, Y. Zhang, S. Liu and J. Xu, J. Mater. Chem. C, 2013, 1, 3376-3390.

7 S. Varughese, J. Mater. Chem. C, 2014, 2, 3499-3516.

8 A. M. Philip, S. K. Manikandan, A. Shaji and M. Hariharan, Chem. - Eur. J., 2018, 24, 18089-18096.

9 B. Shao, R. Jin, A. Li, Y. Liu, B. Li, S. Xu, W. Xu, B. Xu and W. Tian, J. Mater. Chem. C, 2019, 7, 3263-3268.

10 R. Li, S. Xiao, Y. Li, Q. Lin, R. Zhang, J. Zhao, C. Yang, K. Zou, D. Li and T. Yi, Chem. Sci., 2014, 5, 3922-3928.

11 M. Jin, T. Seki and H. Ito, J. Am. Chem. Soc., 2017, 139, 7452-7455.

12 M. Jin, T. Sumitani, H. Sato, T. Seki and H. Ito, J. Am. Chem. Soc., 2018, 140, 2875-2879.

13 H. Yu, X. Song, N. Xie, J. Wang, C. Li and Y. Wang, Adv. Funct. Mater., 2021, 31, 2007511.

14 C. Ge, J. Liu, X. Ye, Q. Han, L. Zhang, S. Cui, Q. Guo, G. Liu, Y. Liu and X. Tao, J. Phys. Chem. C, 2018, 122, 15744-15752.

15 C. Wang and Z. Li, Mater. Chem. Front., 2017, 1, 2174-2194.

16 T. Seki and H. Ito, Chem. - Eur. J., 2016, 22, 4322-4329.

17 G. R. Krishna, M. S. R. N. Kiran, C. L. Fraser, U. Ramamurty and C. M. Reddy, Adv. Funct. Mater., 2013, 23, 1422-1430.

18 G. R. Krishna, R. Devarapalli, R. Prusty, T. Liu, C. L. Fraser, U. Ramamurty and C. M. Reddy, IUCrJ, 2015, 2, 611-619.

19 M. Echeverri, C. Ruiz, S. Gámez-Valenzuela, M. AlonsoNavarro, E. Gutierrez-Puebla, J. L. Serrano, M. C. Ruiz Delgado and B. Gómez-Lor, ACS Appl. Mater. Interfaces, 2020, 12, 10929-10937.

20 Z. Wang, Z. Peng, K. Huang, P. Lu and Y. Wang, J. Mater. Chem. C, 2019, 7, 6706-6713.
21 M. Echeverri, I. Martín, A. Concellón, C. Ruiz, M. S. Anselmo, E. Gutiérrez-Puebla, J. L. Serrano and B. GómezLor, ACS Omega, 2018, 3, 11857-11864.

22 M. Echeverri, C. Ruiz, S. Gámez-Valenzuela, I. Martín, M. C. R. Delgado, E. Gutiérrez-Puebla, M. Á. Monge, L. M. Aguirre-Díaz and B. Gómez-Lor, J. Am. Chem. Soc., 2020, 142, 17147-17155.

23 S. Ito, S. Nagai, T. Ubukata, T. Ueno and H. Uekusa, Cryst. Growth Des., 2020, 20, 4443-4453.

24 J. Chen, D. Li, W. Chi, G. Liu, S. H. Liu, X. Liu, C. Zhang and J. Yin, Chem. - Eur. J., 2018, 24, 3671-3676.

25 P. Gautam, R. Maragani, S. M. Mobin and R. Misra, RSC Adv., 2014, 4, 52526-52529.

26 C. Dou, D. Chen, J. Iqbal, Y. Yuan, H. Zhang and Y. Wang, Langmuir, 2011, 27, 6323-6329.

27 S. Ito, T. Yamada, T. Taguchi, Y. Yamaguchi and M. Asami, Chem. - Asian J., 2016, 11, 1963-1970.

28 C. Zhu, Q. Luo, Y. Shen, C. Lv, S. Zhao, X. Lv, F. Cao, K. Wang, Q. Song, C. Zhang and Y. Zhang, Angew. Chem., Int. Ed., 2021, 8510-8514.

29 E. Xu, H. Zhong, J. Du, D. Zeng, S. Ren, J. Sun and Q. Fang, Dyes Pigm., 2009, 80, 194-198.

30 L. Wang, Y. Zhang, L. Liu and Y. Wang, J. Org. Chem., 2006, 71, 1284-1287.

31 S. Tsuzuki and N. Sato, J. Phys. Chem. B, 2013, 117, 6849-6855.

32 M. R. Ams, N. Trapp, A. Schwab, J. V. Milić and F. Diederich, Chem. - Eur. J., 2019, 25, 323-333.

33 J. Hwang, P. Li, M. D. Smith, C. E. Warden, D. A. Sirianni, E. C. Vik, J. M. Maier, C. J. Yehl, C. D. Sherrill and K. D. Shimizu, J. Am. Chem. Soc., 2018, 140, 13301-13307.

34 A. G. Shtukenberg, Y. O. Punin, A. Gujral and B. Kahr, Angew. Chem., Int. Ed., 2014, 53, 672-699.

35 K. Muthamma, D. Sunil and P. Shetty, Mater. Today Chem., 2020, 18, 100361. 